\title{
El componente liderazgo en la validación de un modelo de gestión escolar hacia la calidad
}

Pablo López Alfaro

Universidad de Chile

\section{Resumo}

A medida que el país alcanza mejor estado de desarrollo, las organizaciones no educacionales han incorporado paulatinamente a su quehacer, elementos de liderazgo, planificación estratégica, de desarrollo organizacional y evaluaciones de la calidad de su gestión, aplicando planes sistemáticos de mejoramiento. Este acento en la gestión también se ha manifestado en el ámbito de las instituciones educativas, siendo el liderazgo uno de los factores de mayor incidencia en la gestión de estas organizaciones.

En este contexto el propósito del presente artículo es dar a conocer la importancia del componente liderazgo en las comunidades escolares en Chile y su relación con la calidad de la educación. El estudio permitió determinar los efectos de variables asociadas a la gestión escolar, entre las que se releva la variable liderazgo, sobre la calidad educativa. Para ello se utilizó una muestra de 870 profesores de centros educativos públicos y privados, a quienes se aplicó una encuesta estructurada. Posteriormente se realizó un exhaustivo análisis psicométrico de los datos, por medio de una serie de análisis exploratorios y confirmatorios que incluyó ecuaciones estructurales.

El estudio pone de manifiesto una realidad en torno a las escuelas como organizaciones. A medida que pasa el tiempo, éstas se transforman en sistemas cada vez más complejos, siendo el liderazgo que se ejerce en un centro educativo una de las variables fundamentales que influye en el logro de los objetivos propuestos por la organización.

\section{Palavras-chave}

Liderazgo - Gestión escolar - Calidad educativa - Modelo causal.

\footnotetext{
Correspondência:

Pablo López Alfaro

Universidad de Chile

Facultad de Ciencias Sociales

Av. Ignacio Carrera Pinto, 1045

Santiago - CHILE

E-mail: pablopez@uchile.cl
} 


\title{
The element of leadership in the validation of a model of quality management for schools
}

\author{
Pablo López Alfaro
}

Universidad de Chile

\begin{abstract}
As a nation achieves higher levels of development, the noneducational organizations gradually incorporate into their tasks elements of leadership, strategic planning, organizational development, and assessments of the quality of their management, applying systematic improvement programs. Such emphasis on the management has also been felt in the realm of educational institutions, with leadership being one of the more recurring factors in the management of these organizations. In this context, the aim of the present article is of revealing the importance of the leadership element in the school communities of Chile, and its relationship with the quality of education. The study has allowed determining the effects of variables associated with school management, chiefly among them the variable of leadership, upon the quality of education. To such end, a structured survey was made with a sample of 870 teachers from public and private education centers. Next, a comprehensive psychometric analysis of the data was carried out through a series of exploratory and confirming analyses that included structural equations. The investigation brings forward the reality of schools as organizations. As time goes by, they are changing into ever more complex systems, in which the leadership exercised at an educational center becomes one of the fundamental variables that determine the success in achieving the objectives proposed by the organization.
\end{abstract}

\section{Keywords}

Leadership - School management - Quality of education Causal model.

Contact:

Pablo López Alfaro

Universidad de Chile

Facultad de Ciencias Sociales

Av. Ignacio Carrera Pinto, 1045

Santiago - CHILE

E-mail: pablopez@uchile.cl 
Diversos estudios nacionales e internacionales han demostrado la importancia de la gestión escolar, y del liderazgo en particular, en los resultados de aprendizaje de los alumnos y en la calidad educativa de los centros. El liderazgo escolar se ha constituido en una prioridad dentro de la política pública diseñada desde el Ministerio de Educación (2005), lo que se ha traducido en potenciar el liderazgo en el sistema educativo, para impulsar el trabajo en equipo y la generación de ambientes de trabajo profesional que permita avanzar en materia de calidad y equidad de la educación. Asimismo, la Fundación Chile (2003) en colaboración con La Pontificia Universidad Católica, instalan un modelo denominado procesual para evaluar y certificar la calidad de la gestión escolar, donde la variable liderazgo es uno de los componentes principales en la explicación de los procesos de calidad.

Coronel (2008), sostiene que el liderazgo es un tema que sigue recibiendo una atención destacada y abundante en trabajos empíricos, reflexiones académicas y publicaciones. No se puede discutir ni poner en duda que estamos frente a un fenómeno real y de gran importancia para el análisis organizativo y el funcionamiento de la vida social. En las organizaciones educativas hablamos de liderazgo pedagógico que nos hace distinguirnos de otros acercamientos y abordajes.

Para Maureira (2008), el estudio del liderazgo aporta un sinnúmero de enfoques y reflexiones en torno a este fenómeno, haciéndose notar como un tema ampliamente estudiado, sin embargo, algunos autores, lo califican como el peor comprendido, dada la complejidad del tema a nivel de la organización escolar, donde el énfasis del trabajo se fundamenta esencialmente en la interacción de distintos agentes educativos: alumnos, profesores, directivos y otros.

\section{Gestión y calidad}

El concepto de calidad está presente en todos los ámbitos de la vida. Aparece asociado a aquello que queremos destacar como atractivo o valioso, a la excelencia, al trabajo bien hecho o a aquello que se quiere reconocer, Marchesi y Martin (1998) la conciben como "un anhelo, un deseo de perfección, un objetivo al cual aproximarse pero que nunca se consigue del todo" (p. 10). Ambas definiciones parecen subrayar los elementos objetivos del concepto, olvidando que éste tiene además un elemento subjetivo: se fundamenta en las necesidades del beneficiario, por lo que los parámetros para medir calidad no están asociados sólo con las características intrínsecas de un producto o servicio.

El concepto ha evolucionado desde un enfoque centrado en las características objetivas del producto o servicio a un enfoque que incorpora elementos de subjetividad, lo cual hace al concepto de calidad alejarse de la consideración del costo como su fundamento y lo convierte en un concepto dinámico, llegando en la actualidad a referirnos permanentemente a gestión de calidad o gestión bien hecha.

La gestión de calidad se basa en un modelo que hace uso intensivo de las personas y no en el capital físico o financiero de una organización, de tal manera que interesa por sobremanera la calidad de las personas que pertenecen a la organización, su integridad, así como la de quienes lideran los procesos. Esta perspectiva obliga a enfatizar la necesidad de las organizaciones no sólo de lanzar un buen producto al exterior, sino además funcionar bien internamente, es decir, no es posible que una organización orientada hacia el beneficiario no esté orientada hacia las personas. Si se logra un buen funcionamiento interno y que los trabajadores se sientan bien tratados y a gusto en la organización, éstos querrán agradar a los benefactores. Albrecht (1995), señala que "Los empleados descontentos son terroristas. Se lo propongan o no, destruyen la calidad del servicio desde la base" (p. 90).

El concepto de calidad ha evolucionado, desde una visión tradicional como plantea Cantón; Gaziel; Warnet (2000), a una visión transformadora. En la primera de ellas la organización actuaba sobre los efectos, con un control de calidad a posteriori, el campo de 
acción estaba limitado a la calidad del producto y se pensaba que era normal que algunos productos fuesen defectuosos, porque la calidad era costosa. La organización estaba centrada en los problemas, no en los beneficiarios a quienes se les intentaba justificar los defectos en el servicio o producto.

La visión transformadora de la calidad, que la acerca al concepto de gestión de calidad, se centra en un control a priori para evitar o prevenir defectos. Su campo de acción es en toda la organización y su gestión está basada en una investigación permanente del usuario, lo que lleva a la organización a estar centrada en la satisfacción del beneficiario, en conocer sus necesidades. La gestión de calidad se basa en principios que incluyen su consideración no como un problema aislado, sino abarcando toda la organización y orientando la gestión hacia el beneficiario externo, el cual es estimado como lo más importante, sin dejar de lado al usuario interno cuyo bienestar es determinante en los resultados de la gestión de calidad.

Bajo esta conceptualización la calidad está modelando la gestión organizacional en todos sus ámbitos constituyendo un marco regulatorio del quehacer institucional que al igual que los conceptos de liderazgo, planificación y visión imprimen direccionalidad a la gestión, enmarcan la acción de los componentes de la organización y constituyen partes de un engranaje donde la gestión juega un rol articulador de estos elementos que moviliza a la organización hacia el cumplimiento de su propósito.

\section{Gestión y liderazgo}

Necesariamente el liderazgo está vinculado al desarrollo de una estrategia para el logro de una visión y la gestión persigue ejecutar esta estrategia lo más eficientemente posible, de tal manera apoyar el proceso de logro de la visión organizacional. Ambos conceptos por lo tanto se asocian estrechamente. Beckhard y Pritchard (2000), sostienen que la visión constituye, un marco en el cual se desarrolla la gestión de la organización,
Un cuadro de un estado futuro para la organización, una descripción de lo que podría ser dentro de algunos años. Es un cuadro dinámico de la organización en el futuro, tal como la ven los directivos. Es más que un sueño o un conjunto de esperanzas, porque la alta administración está evidentemente comprometida a realizarla: es un compromiso. (p. 27).

Para Senge (1992), el compromiso que se deriva de una visión compartida impacta en toda la organización y genera una fuerza de cambio en su interior que obliga a definir un conjunto de procesos para avanzar en su consecución. En definitiva produce una presión sobre la gestión para que ésta sea lo suficientemente efectiva para alcanzarla.

Una visión compartida no es una idea. $\mathrm{Ni}$ siquiera es una idea tan importante como la libertad. Es una fuerza en el corazón de la gente, una fuerza de impresionante poder. Puede estar inspirada por una idea, pero si es tan convincente como para lograr el respaldo de más de una persona cesa de ser una abstracción. Es palpable. La gente comienza a verla como si existiera. (p. 261).

Desde esta perspectiva, podemos concluir que la visión es uno de los determinantes básicos de la gestión organizacional, ya que no sólo le imprime dirección, sino que además modela los procesos que se requiera ejecutar para su logro, los determina y valida como efectivos en la medida que sirven a sus propósitos. El rol del liderazgo en este proceso es fundamental. Lepeley (2001), concibe el liderazgo como "la ciencia de conducir a una organización de la situación actual a una situación futura comparativamente mejor, donde las personas son el principal factor de cambio" (p. 23), se relaciona así ampliamente con el concepto de gestión, no obstante la diferencia entre ambos conceptos.

El liderazgo, como hecho social y como concepto científico, sigue navegando en un mar de imprecisiones, no sólo por la amplitud de su 
contenido, sino también por la variedad de perspectivas desde donde se le ha enfocado, lo que lo ha llevado a evolucionar desde un enfoque netamente psicológico hasta una perspectiva de interacción social y a mantener latente su relación con autoridad y poder, llevando incluso a generar imprecisiones entre los tres conceptos.

Ruiz (1995), entiende por liderazgo "el arte de conseguir que las personas cumplan una tarea voluntariamente" definición que asociada a lo planteado por el mismo autor, que señala que "el liderazgo equivale al ejercicio de la autoridad que consigue una obediencia entusiasta de parte de sus súbditos” (p. 371), nos llevan a reflexionar respecto del sentido que el liderazgo tiene para una organización.

Difícilmente una organización puede desarrollar una buena gestión si no mantiene un liderazgo efectivo que la haga trascender desde un plano operativo, del día a día, a uno de más amplio plazo relacionado con su desarrollo. Resulta esencial establecer que mientras el liderazgo está vinculado estrechamente al desarrollo de una visión y estrategias, la gestión se encuentra asociada a la manutención del funcionamiento de un sistema, relacionándose, más bien con la efectividad de la realización de las tareas, sostenida por una estructura organizativa y con funciones administrativas como la planificación, organización y control, que con la dirección.

Para algunos autores, como López Ruperez (2001), el modelo en esta dimensión incluye la consideración de cómo el liderazgo del equipo directivo inspira y conduce hacia la calidad total, dentro de un proceso que se considera fundamental para la organización y que se entiende como de mejora continua. El liderazgo del equipo directivo, es conceptualizado como:

El compromiso efectivo de los cuadros directivos de la organización y del director general con la calidad, que en la práctica significa la definición de los objetivos de calidad por parte del máximo nivel ejecutivo y orientar a toda la organización en ese sentido. (p. 48).
Cantón; Gazïel; Warnet (2000), considera al Liderazgo como un criterio a evaluar, un agente facilitador del proceso y lo define como:

El comportamiento y actuación del Equipo Directivo y del resto de los responsables para guiar a la organización hacia la calidad. A través de él, se pretende evaluar cómo aquellos que tienen alguna responsabilidad en la organización estimulan, apoyan y fomentan la gestión de calidad, como un proceso de mejora continua” (p. 188).

Una de las características más relevantes del liderazgo, desde la perspectiva de una gestión de calidad, es la capacidad de generar ambientes al interior de la organización que alienten la innovación, de tal manera que todos sus miembros se sientan motivados a aportar ideas para el mejoramiento continuo en todos los ámbitos de la gestión. En este contexto, resulta necesario detenernos en una de las teorías del liderazgo, que apunta al logro de este objetivo: La teoría del Liderazgo Transformacional.

Al respecto, Geijsel, Sleegers y Van Den Berg (2000), presentan investigaciones de liderazgo transformacional en las innovaciones a gran escala, donde se describe que las tres dimensiones de liderazgo más importantes son: la visión, la consideración individual y la estimulación intelectual. Se espera que estas tres dimensiones de liderazgo transformacional tengan un impacto positivo sobre la implementación de las innovaciones por parte de los profesores vía su influencia en la participación en la toma de decisiones, sentimientos de incertidumbre y actividades de desarrollo profesional.

\section{Modelos de gestión de la calidad}

La formalización de los principios de la gestión en modelos, es posible ya que a lo largo de la historia se identifica un conjunto de marcos conceptuales, técnicos e instrumentales que ha ido orientando el cambio organizacional, marcos que poseen características claramente 
distintivas, representando cada uno de ellos, según Casassus (2000),

Una forma de respuesta a limitaciones que presenta el modelo anterior o a situaciones restrictivas del entorno de los modelos anteriores. Cada nuevo marco conceptual no invalida al anterior, pero si representa un avance con respecto a él, generando una acumulación teórica e instrumental. (p. 4)

Para la elaboración del modelo teórico a partir del cual se diseñaron los instrumentos de recolección de información se consideraron dos trabajos. El primero corresponde al Modelo Europeo EFQM, Fundación Europea para la Gestión de Calidad (1999), y el segundo correspondió al realizado por el Ministerio de Educación (2005) en el desarrollo del modelo SACGE.

\section{EI modelo europeo EFQM (European Foundation for Quality Management)}

La utilización del modelo EFQM como referencia se debe a que facilita un diagnóstico sistemático de todas las áreas de la organización y suministra reglas de análisis ampliamente validadas por la experiencia. El 21 de abril de 1999 se anunció la última versión, que se adap- tó para su aplicación a los centros educativos en el año 2001. La misión de la EFQM es:

- Estimular y ayudar a las organizaciones a participar en actividades de mejora que las lleven, en última instancia, a la excelencia en la satisfacción de sus clientes y de sus empleados, en su impacto social y en sus resultados.

- Apoyar a los directivos de las organizaciones en la aceleración del proceso de convertir la Gestión de Calidad Total en un factor decisivo para conseguir una posición de competitividad global.

\section{Dimensiones aportadas por el modelo EFQM}

El modelo está compuesto por nueve dimensiones, estructuradas en dos bloques: el de los agentes o facilitadores, cuyo peso total es del 50\% de la puntuación global, y el de los resultados, con el otro 50\%, es decir con 500 puntos de los 1000 que componen el modelo.

\section{Los agentes o facilitadores}

El modelo incluye los siguientes agentes o facilitadores: el liderazgo, la gestión del personal, la política y la estrategia, los recursos y los procesos:

Figura 1 - Modelo Europeo de Gestión de Calidad (adaptado a la Educación). Pérez Juste (2001, p. 37).

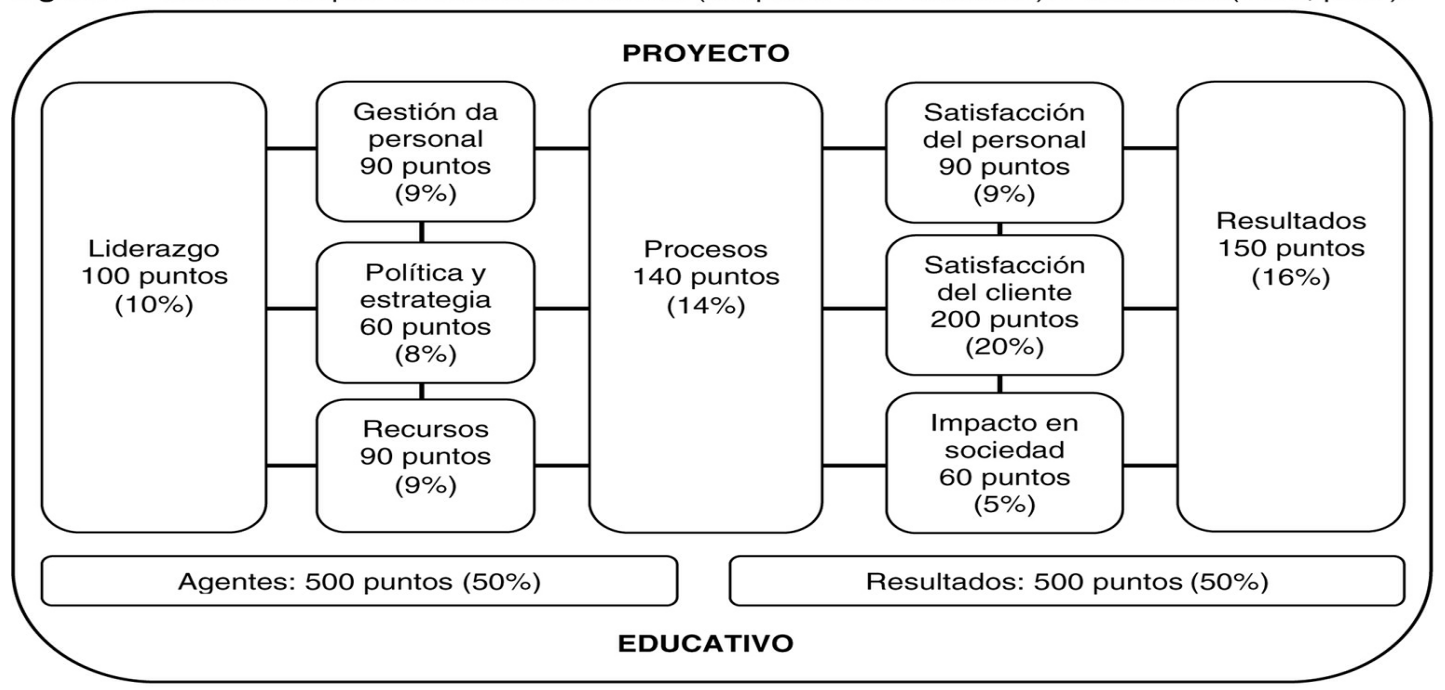


1. Liderazgo: comportamiento y actuación del Equipo Directivo y del resto de los responsables para guiar a la organización hacia la calidad. Por medio de él, se pretende evaluar cómo aquellos que tienen alguna responsabilidad en la organización estimulan, apoyan y fomentan la gestión de calidad, como un proceso de mejora continua (100 puntos, 10\%).

2. Política y Estrategia: es concebida como un instrumento de creación, diagnóstico, análisis, reflexión y toma de decisiones colectivas en torno al desarrollo de la misión institucional y el camino a seguir para conseguirla optimizando el potencial de las personas y la utilización de recursos disponibles, adecuándose con rapidez a los cambios para exceder en la satisfacción de demandas de los beneficiarios (80 puntos, 8\%)

3. Gestión del Personal: se refiere a la forma en que la organización logra que su personal participe en forma activa en un proceso de mejoramiento continuo, lo cual deriva en el aprovechamiento de las capacidades de cada persona dentro de la organización en pos del logro de los objetivos institucionales y de acuerdo a la optimización de su bienestar o satisfacción (90 puntos, 9\%).

4. Recursos: en el modelo, se consideran los sistemas de asignación y de gestión de los recursos materiales, tecnológicos, financieros y de información de acuerdo a las políticas y estrategias en pos del mejoramiento progresivo de la calidad (90 puntos, 9\%).

5. Procesos: esta dimensión se relaciona con la forma en que la organización identifica, revisa y mejora los procesos clave o críticos y las actividades que se requieren para cumplir sus fines, en un marco definido por los estándares de calidad establecidos (140 puntos, 14\%).

\section{Los resultados}

El modelo EFQM considera cuatro dimensiones: satisfacción del personal, satisfacción del cliente, impacto de la sociedad y resultados.
6. Satisfacción del personal: esta dimensión apunta hacia la necesidad de mantener un sistema de indicadores de los niveles de satisfacción del personal, el grado en el que sus necesidades y expectativas están siendo satisfechas (90 puntos, 9\%).

7. Satisfacción del cliente: en este criterio se conjuga tanto la percepción manifestada por las persona externas sobre la organización, sus productos, y las relaciones, como las valoraciones que realizan los evaluadores de las medidas destinadas a satisfacer a los beneficiarios. Aspectos como la accesibilidad, el apoyo técnico, el trato recibido en las reclamaciones o los índices de rechazo son los aspectos que toman en consideración (200 puntos, 20\%).

8. Impacto en la Sociedad: abarca a la sociedad en su conjunto, en las conexiones indirectas o menos directas que la organización mantiene con ella (60 puntos, 6\%).

9. Resultados: servicios prestados, especialmente aquellos relacionados con la naturaleza de la organización. Esto significa que se espera lograr resultados en diversos ámbitos, desde aquellos derivados de la misión institucional, las metas establecidas en relación con la política, estrategia y objetivos derivados de sus planes de mejora hasta aquellos derivados de los indicadores de rendimiento que son valorados (150 puntos, $15 \%$ ).

Pérez Juste (2001), plantea que las particularidades de la realidad educativa no quedan, a su juicio, suficientemente recogidas en el Modelo Europeo. Todos los criterios, debieran estar basados en o impregnados por el Proyecto Educativo de la organización.

Así contextualizado, el proyecto educativo constituye una dimensión trascendental en el modelo EFQM, en su adaptación a la educación y se define como la base de un modelo de gestión de calidad de una organización educativa, en torno al cual ésta se estructura y se evalúa de acuerdo al grado en que todas las otras dimensiones, que no son puestas en duda, aportan al logro del proyecto educativo. 
Figura 2 - Modelo de Calidad de la Gestión Escolar - Sistema de aseguramiento de la Calida de la Gestión Escolar. Ministerio de Educación, Chile, 2005, p. 16.

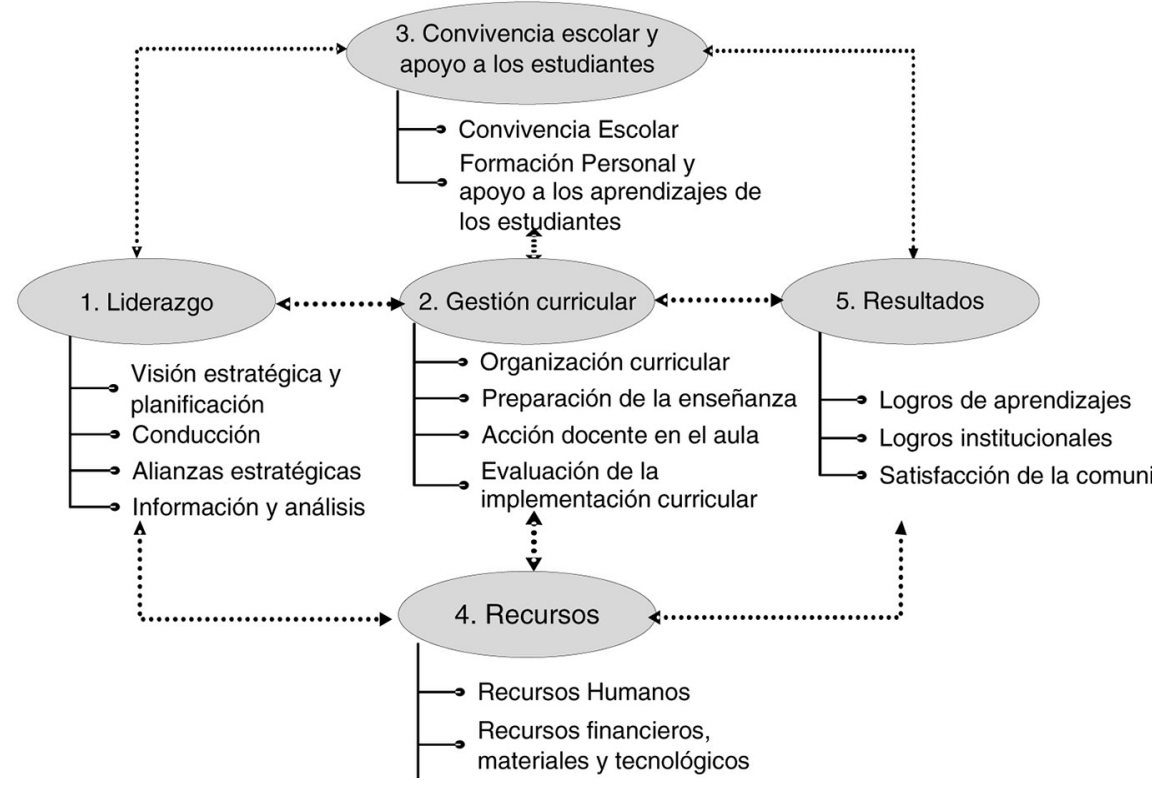

\section{Modelo de calidad de la gestión escolar}

En los últimos años en la realidad chilena, también se han venido incorporando los aportes de modelos de gestión de calidad. Uno de ellos es el desarrollado por el Ministerio de Educación, denominado SACGE (Sistema de aseguramiento de la Calidad de la Gestión Escolar) conformado por cinco elementos de gestión: Liderazgo, Gestión curricular, Convivencia escolar, Recursos y Resultados.

El eje central del Sistema de Aseguramiento de la Calidad de la Gestión escolar es el Mejoramiento continuo que consiste en una revisión sistemática y permanente de los procesos de los establecimientos, para luego formular acciones de mejoramiento que permita obtener logros de aprendizaje para todos los alumnos.

A partir de estos trabajos, en esta investigación se propone un modelo teórico que da cuenta de siete dimensiones que explican la calidad de los centros educativos y que corresponden a: a) Liderazgo escolar; b) Planificación y estrategia; c) Gestión de recursos; d) Gestión de personas; e) Procesos; f) Satisfacción y g) Resultados.

\section{Metodología del estudio}

Este estudio presenta la evaluación del componente liderazgo en la gestión escolar de setenta establecimientos educativos de Enseñanza Básica ${ }^{1}$ y Media $^{2}$ por la relevancia que tiene en la calidad educativa. Se realizaron análisis factoriales: exploratorio, confirmatorio y análisis causal utilizando ecuaciones estructurales.

El modelado con ecuaciones estructurales incluye dos submodelos (Cea, 2002):

a) El modelo estructural: nos permitió describir las relaciones causales entre variables latentes ${ }^{3}$ exógenas ${ }^{4}$ y endógenas ${ }^{5}$ mediante ecuaciones estructurales lineales, que expresan la estructura causal asumida entre las variables.

b) En el modelo de medición se establecieron las relaciones de las variables latentes con sus indicadores.

1. La Enseñanza Básica o Primaria en Chile corresponde al ciclo inicial de los estudios escolares cuya duración es de 8 años divididos en dos ciclos y ocho grados (de 6 a 13 años de edad ideal).

2. La Enseñanza Secundaria o Media se compone de 4 niveles 0 cursos, comienza en $1^{\circ}$ medio (14-15 años) y finaliza con $4^{\circ}$ medio (17-18 años), existen dos modalidades de enseñanza media en Chile la "Científica Humanista y la Técnico Profesional". 
De acuerdo con investigaciones empíricas y consideraciones teóricas planteamos el siguiente modelo (proceso - producto) como consecuencia de la interacción de los comportamientos de docentes y estudiantes.

Las variables de gestión escolar se recogieron por medio de la encuesta diseñada, cuya validación empírica se realizó utilizando ecuaciones estructurales con variables latentes; las que fueron modeladas utilizando el software LISREL (Linear Structural Relations) (Jöreskog; Sörbom, 1993).

Las dimensiones y variables del instrumento se muestran en la Tabla 2. Los descriptores de cada variable fueron extraídos desde el Modelo Europeo de Calidad EFQM, y del modelo desarrollado por el Ministerio de Educación (2005) denominado SACGE.

Tabla 1 - Modelo proceso-producto, el liderazgo como componente del modelo gestión hacia la calidad educativa.

\begin{tabular}{|l|l|}
\hline \multicolumn{1}{|c|}{ Proceso } \\
\hline Liderazgo educativo \\
\hline Planificación y estrategia \\
\hline Gestión de recursos \\
\hline Procesos \\
\hline Gestión de personas \\
\hline Satisfacción \\
\hline Resultados \\
\hline
\end{tabular}

La muestra del estudio estuvo compuesta por 70 establecimientos de las regiones ${ }^{6} \mathrm{~V}$, VIII y Región Metropolitana. De ellos según dependencia administrativa $^{7}, 32$ eran municipales (45,7\%), 23 particulares subvencionados $(32,8 \%)$ y 15 particulares pagados $(21,4 \%)$. Los instrumentos (Escala tipo Likert) desarrollados y validados fueron aplicados a una muestra de 870 profesores y directivos trabajando con una base de 60.000 datos.

Tabla 2 - Dimensión liderazgo y otras variables asociadas a la gestión escolar (Elaboración propia).

\begin{tabular}{|l|l|l|l}
\hline Dimensiones & \multicolumn{1}{|c|}{ Variables } & Dimensiones & \multicolumn{1}{c}{ Variables } \\
\hline Liderazgo & $\begin{array}{l}\text { Compromiso } \\
\text { Reconocimiento }\end{array}$ & Satisfacción & $\begin{array}{l}\text { Nivel de agrado con } \\
\text { la gestión y } \\
\text { funcionamiento del } \\
\text { establecimiento }\end{array}$ \\
\hline $\begin{array}{l}\text { Planificación } \\
\text { y estrategia }\end{array}$ & $\begin{array}{l}\text { Desarrollo de } \\
\text { la estrategia } \\
\text { Comunicación }\end{array}$ & Resultados & $\begin{array}{l}\text { Logros del } \\
\text { establecimiento en } \\
\text { todos sus ámbitos de } \\
\text { gestión }\end{array}$ \\
\hline $\begin{array}{l}\text { Gestión de } \\
\text { personas }\end{array}$ & $\begin{array}{l}\text { Competencias } \\
\text { Motivación }\end{array}$ & $\begin{array}{l}\text { Calidad } \\
\text { Educativa }\end{array}$ & $\begin{array}{l}\text { Expectativas } \\
\text { Percepciones de los } \\
\text { docentes en los } \\
\text { ámbitos de gestión }\end{array}$ \\
\hline Recursos & $\begin{array}{l}\text { Gestión de } \\
\text { recursos } \\
\text { Innovación }\end{array}$ & \multicolumn{2}{|l}{} \\
\hline
\end{tabular}

Procedimientos: la primera tarea consistió en desarrollar los estadísticos descriptivos, análisis de fiabilidad y análisis factoriales exploratorios.

En la segunda etapa se realizaron los análisis factoriales confirmatorios de ambos modelos de medición, tanto de variables latentes exógenas (independientes) como endógenas (dependientes) definidas en el modelo.

En la tercera etapa se aplicaron los sistemas de ecuaciones estructurales en la validación del modelo causal, en conjunto con el modelo estructural y de medición.

\section{Resultados}

Los resultados que se presentan a continuación, corresponden a análisis que se focalizan en aquellos datos que acentúan la gestión escolar y variables asociadas destacando la dimensión liderazgo escolar y su relación con la calidad educativa en los establecimientos primarios y secundarios que se incluyeron en el estudio.

\section{Estudio 1:}

Análisis de la confiabilidad del instrumento

Los cálculos de los coeficientes de frabilidad de las dimensiones originales del instrumento adoptados de los modelos SACGE y EFQM y conformados por las variables: Liderazgo, Planificación, Gestión de Recursos, Personas, Procesos, Satisfacción, Resultados y Calidad Educativa, nos indicaron que la escala general muestra niveles de consistencia interna ( $\alpha$

3. Constuctos hipotéticos no observables directamente, pero se estiman por medio de indicadores o variables observables.

4. Variables independientes que no son explicadas por otras variables del modelo. Su variabilidad se debe a causas externas al modelo.

5. Corresponde a las variables dependientes explicadas por las variables independientes del modelo.

6. Chile está dividido políticamente en regiones, en la actualidad el total es de 15. Las Regiones en Chile son las subdivisiones territoriales superiores del país, sobre las provincias y superiores a las comunas.

7. Al inicio de la década de 1980 se produce un cambio estructural de las bases de la educación en Chile. Es entonces cuando los establecimientos educacionales que dependían del Ministerio de Educación son traspasados a la administración municipal, se crean además los colegios particulares subvencionados, que son financiados por el estado y administrados por privados, manteniéndose la existencia de colegios particulares pagados que son financiados en un $100 \%$ por los padres. 
= 0.96). De manera similar constatamos una alta consistencia interna en la medida para cada una de las variables asociadas con la gestión escolar.

Tabla 3 - Resultados de confiabilidad referida a variables asociadas a la gestión escolar.

\begin{tabular}{|l|c|c|}
\hline \multicolumn{1}{|c|}{ Dimensión teórica } & Valor “. ” & $\begin{array}{c}\text { Número de } \\
\text { ítem s }\end{array}$ \\
\hline Liderazgo & 0.88 & 9 \\
\hline Planificación y Estrategia & 0.88 & 7 \\
\hline Personas & 0.87 & 11 \\
\hline Recursos & 0.77 & 5 \\
\hline Procesos & 0.85 & 8 \\
\hline Satisfacción & 0.90 & 9 \\
\hline Resultados & 0.84 & 7 \\
\hline Calidad Educativa & 0.72 & 4 \\
\hline Instrumento completo & $\mathbf{0 . 9 6}$ & $\mathbf{6 0}$ \\
\hline
\end{tabular}

Análisis factorial exploratorio ${ }^{8}$ (AFE) para variables latentes exógenas $\mathrm{o}$ independientes

La matriz de correlaciones entre las variables de liderazgo, planificación, personas, recursos y procesos, entrega valores altos que superan 0,30 en valor absoluto, lo que nos señala la conveniencia de proseguir con la factorización.

El índice (KM0: Kaiser-Meyer-Olkin ${ }^{9}$ ) que muestra la existencia de intercorrelación entre variables, presenta un valor de 0,916, el cual es bueno y ratifica la decisión de agrupación de variables en factores comunes.

Con el fin de comprobar empíricamente la estructura propuesta, y la organización para las variables relacionadas en el modelo, y dado que ésta es una fase exploratoria, se observó la presencia de cinco factores (Tabla 4): Liderazgo, Planificación, Personas, Recursos y Procesos, que en conjunto explican el 58\% de la varianza de los datos.

Análisis factorial exploratorio, variables latentes endógenas o dependientes

Los análisis de la matriz de correlaciones entre las variables de satisfacción, resultados y calidad educativa, presentan valores altos que superan 0,34 en valor absoluto, referencia que nos señala la conveniencia de seguir con la factorización.

El índice (KMO: Kaiser-Meyer-Olkin) presenta un indicador de 0.944, el cual es bueno y confirma la decisión de agrupar variables en factores comunes.
Tabla 4 - Matriz de componentes, variables: liderazgo, planificación, personas, recursos y procesos.

\begin{tabular}{|l|c|c|c|c|c|}
\cline { 2 - 6 } \multicolumn{1}{c|}{} & \multicolumn{5}{c|}{ Componente } \\
\cline { 2 - 6 } \multicolumn{1}{c|}{} & $\mathbf{1}$ & $\mathbf{2}$ & $\mathbf{3}$ & $\mathbf{4}$ & $\mathbf{5}$ \\
\hline Lider5 & .625 & & & & \\
Lider7 & .616 & & & & \\
& .743 & & & & \\
Plani11 & & .565 & & & \\
Perso18 & & .568 & & & \\
Perso19 & & & .802 & & \\
Recur28 & & & .836 & & \\
Recur31 & & & & .781 & \\
Proce37 & & & & .789 & \\
Proce38 & & & & & .828 \\
\hline
\end{tabular}

En relación a la extracción de componentes principales, se observó la presencia de tres factores que en conjunto explican el $60 \%$ de la varianza (satisfacción, resultados y calidad educativa).

El análisis factorial exploratorio tanto para variables latentes exógenas como endógenas permitió determinar que, la agrupación de variables se corresponde con los planteamientos teóricos, para luego desarrollar análisis posteriores con fines confirmatorios. (Hair et al., 2001).

Finalmente, es importante consignar que para ambos tipos de variables (exógenas y endógenas), el análisis factorial exploratorio proporciona sólo un criterio matemático, orientándonos hacia la validación de los modelos de medida para las distintas variables que forman parte de nuestro estudio.

Tabla 5 - Matriz de componentes, variables: satisfacción, resultados y calidad.

\begin{tabular}{|l|c|c|c|}
\cline { 2 - 4 } \multicolumn{1}{c|}{} & \multicolumn{3}{c|}{ Componente } \\
\cline { 2 - 4 } \multicolumn{1}{c|}{} & $\mathbf{1}$ & $\mathbf{2}$ & $\mathbf{3}$ \\
\hline Satis45 & .735 & & \\
Satis48 & .816 & & \\
Satis49 & .849 & & \\
Resul54 & & .666 & \\
Resul55 & & .676 & \\
Resul56 & & .448 & \\
Calid57 & & & .896 \\
Calid59 & & & .670 \\
\hline
\end{tabular}

8. Por medio del AFE se analiza la relación mutua entre diversas variables para determinar el grado en que se justifica cada variable por cada factor. Uno de sus objetivos fundamentales es analizar la correlación existente en una serie de variables con el propósito de descubrir si comparten alguna estructura latente.

$\mathbf{9 .}$ Índice que compara las correlaciones observadas con sus correspondientes correlaciones múltiples. La finalidad es determinar la propiedad de hacer un análisis factorial. 
Estudio 2:

Análisis factorial confirmatorio ${ }^{10}$ (AFC), para ambos modelos de medición utilizando el método de ecuaciones estructurales

a) Para el modelo de medición de variables latentes exógenas se logra determinar la validez de los indicadores como medidas de las dimensiones de liderazgo, planificación, personas, recursos y procesos.

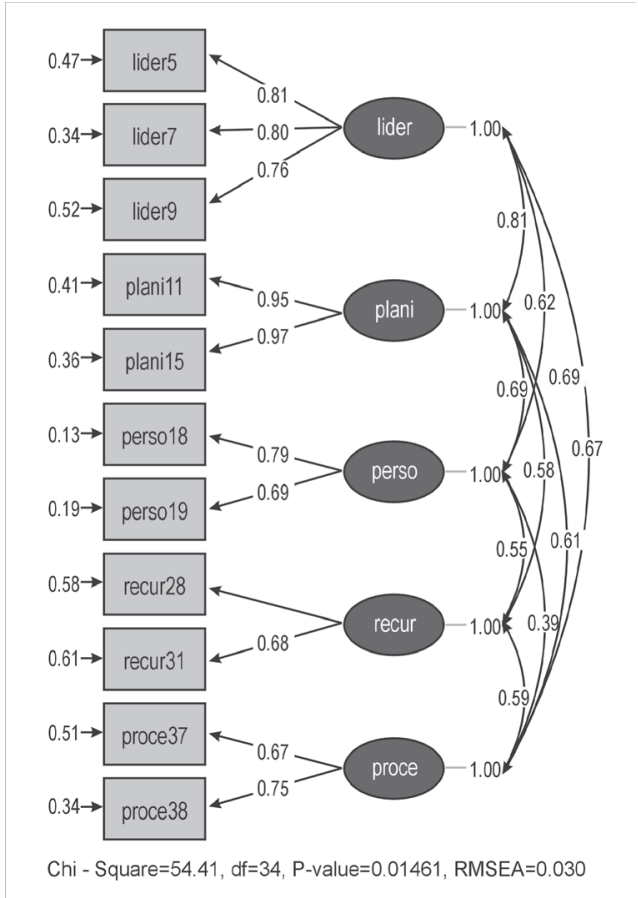

Figura 3: Modelo de medición variables latentes exógenas o independientes

Índices de bondad de ajuste para el diagnóstico global.

\begin{tabular}{|c|c|c|c|c|}
\hline CFI & NFI & GFI & RMSEA & $\chi^{2}$ Normado \\
\hline .995 & .993 & .987 & .0030 & 1,44 \\
\hline
\end{tabular}

Se observa que el ajuste es positivo en la totalidad de los índices obtenidos para el modelo que agrupa en cinco factores las once variables. En este sentido, los índices
CFI (Comparative Fit Index) y NFI (Normal Fit Index) propuestos por (Bentler; Dudgeon, 1996), y el presentado por (Jöreskog; Sörbom, 1989) GFI (Goodness of fit index), muestran valores por encima de 0.90 los cuales son considerados muy buenos mientras que $\chi 2$ Normado (Jöreskog, 1969) presenta un valor de 1,44 que se puede interpretar como un buen ajuste; lo mismo ocurre para RMSEA (Root Mean Square Error of Approximation) que resulta significativo con valores menores a 0.05 (Brown; Kudeck, 1993).

En conjunto, los resultados obtenidos por medio de los análisis factoriales confirmatorios informan de la validez de la estructura factorial hipotetizada. Es decir se confirma la existencia de un modelo de once variables agrupadas en cinco factores principales: liderazgo, planificación, recursos, personas, procesos.

b) En relación al modelo de medición de variables latentes endógenas determinamos la validez de nuestros indicadores como medidas de las dimensiones de satisfacción, resultados y calidad educativa.

Índices de bondad de ajuste para el diagnóstico global

\begin{tabular}{|l|l|l|l|l|}
\hline CFI & NFI & GFI & RMSEA & $\chi^{2}$ Normado \\
\hline .987 & .982 & .982 & .048 & 3,88 \\
\hline
\end{tabular}

El análisis realizado es similar al anterior, obteniéndose un ajuste positivo en los índices del modelo que agrupa en tres factores las siete variables, excepto $\chi^{2}$ Normado, presenta un valor de 3,88 que se puede interpretar como un regular ajuste.

Los resultados en el estudio 2, de ambos análisis factoriales confirmatorios informan de la validez de la estructura factorial hipotetizada. Es decir se confirma la existencia de un modelo de once variables agrupadas en cinco factores

10. Por medio del AFC se estima la relación entre los conceptos que se está estudiando (liderazgo, planificación, personas, recursos, procesos, satisfacción, resultados y calidad educativa) y los indicadores utilizados para medirlos. 
principales: liderazgo, planificación, recursos, personas, procesos y otro de siete variables agrupadas en tres factores principales: satisfacción, resultados y calidad educativa.

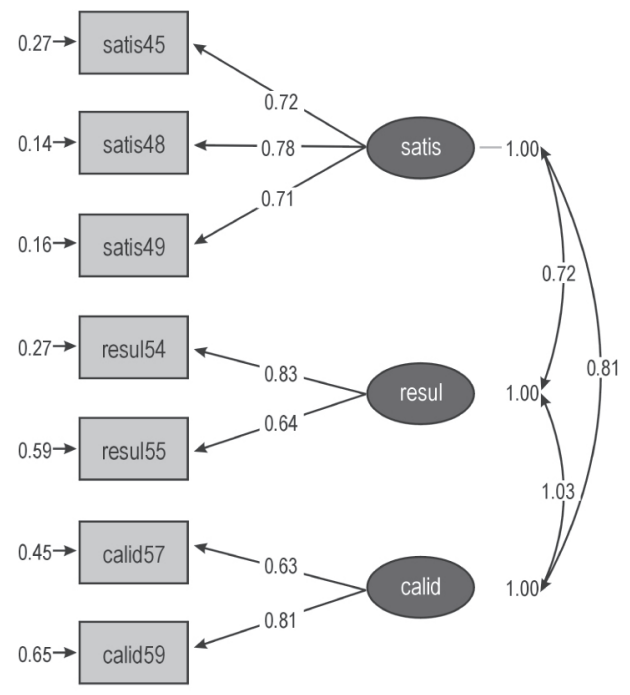

Chi - Square $=24.82, \mathrm{df}=10, \mathrm{P}$-value $=0.00569, \mathrm{RMSEA}=0.084$

Figura 4: Modelo de medición de variables latentes endógenas o dependientes.

Estudio 3:

\section{Estimación del modelo de relaciones con ecuaciones estructurales ${ }^{11}$}

a) Análisis empíricos iniciales de los modelos de medición y estructural, tanto para las variables latentes exógenas como endógenas especificadas en el modelo causal, tal como se muestra en el diagrama. (Figura 5)

En un primer estudio, la información que entrega LISREL en relación a los índices de bondad de ajuste, a los coeficientes estimados en los modelos de medida y estructural exceden los límites aceptables. Por ejemplo, i) el valor de $\chi 2(1431,21)$ del modelo planteado en la fig. 5, no es aceptable ya que el nivel de significación estadística ( $p=0,000)$, no es mayor que 0.05 ó 0.01 , por lo que las matrices de entrada prevista y efectivas son diferentes; ii) el intervalo de confianza para el error cuadrático de aproximación (RMSEA) cuyo valor de 0.135, es muy superior a 0.05 lo que conduce a rechazar un error de marginalidad de la hipótesis para un ajuste aceptable.

Un segundo análisis es la búsqueda de estimaciones infractoras ${ }^{12}$, hallándose varianzas de error negativa, coeficientes estandarizados que exceden o están muy cerca de 1.0 o errores estándar muy elevados. Con este análisis fue necesario eliminar los indicadores plani11, plani15, perso19, satis48, resul54 y resul56, por lo que habrá que reespecificar el modelo las veces que sea necesario hasta encontrar el que mejor se ajuste a los datos.

b) Modelo final (Figura 6). A partir de los análisis desarrollados, presentamos el diagrama del modelo final resultante y sus correspondientes índices de bondad de ajuste.

Respecto del modelo final, un primer análisis de la información que entrega LISREL en relación a los índices de bondad de ajuste, es la siguiente: a) el estadístico $\chi 2$ del modelo final modificado nos entrega un valor marginal aceptable. Su valor es de 81.37 con $\rho=0.00101$. b) el intervalo de confianza para el error cuadrático de aproximación (RMSEA) presenta un valor de 0.034 , que es menor a 0.05 , lo que conduce a aceptar el error de marginalidad de la hipótesis para un buen ajuste.

Un segundo análisis es la búsqueda de estimaciones infractoras ${ }^{12}$. En este análisis no se encontraron varianzas de error negativa, coeficientes estandarizados ni tampoco errores estándar elevados que excedieran los límites aceptables.

Finalmente se realizaron análisis de otros índices de bondad de ajuste del modelo final para el diagnóstico global. Este análisis, permitió confirmar el grado de ajuste entre el modelo y los datos.

11. Los modelos de ecuaciones estructurales son sistemas de variables cuya organización, distribución y relación se establece previamente a partir de una serie de hipótesis. Se caracterizan por dos componentes: 1) el modelo estructural que relaciona variables latentes exógenas y endógenas y 2) el modelo de medida que permite usar variables observadas o indicadores para la medida de una variable latente exógena o endógena. 


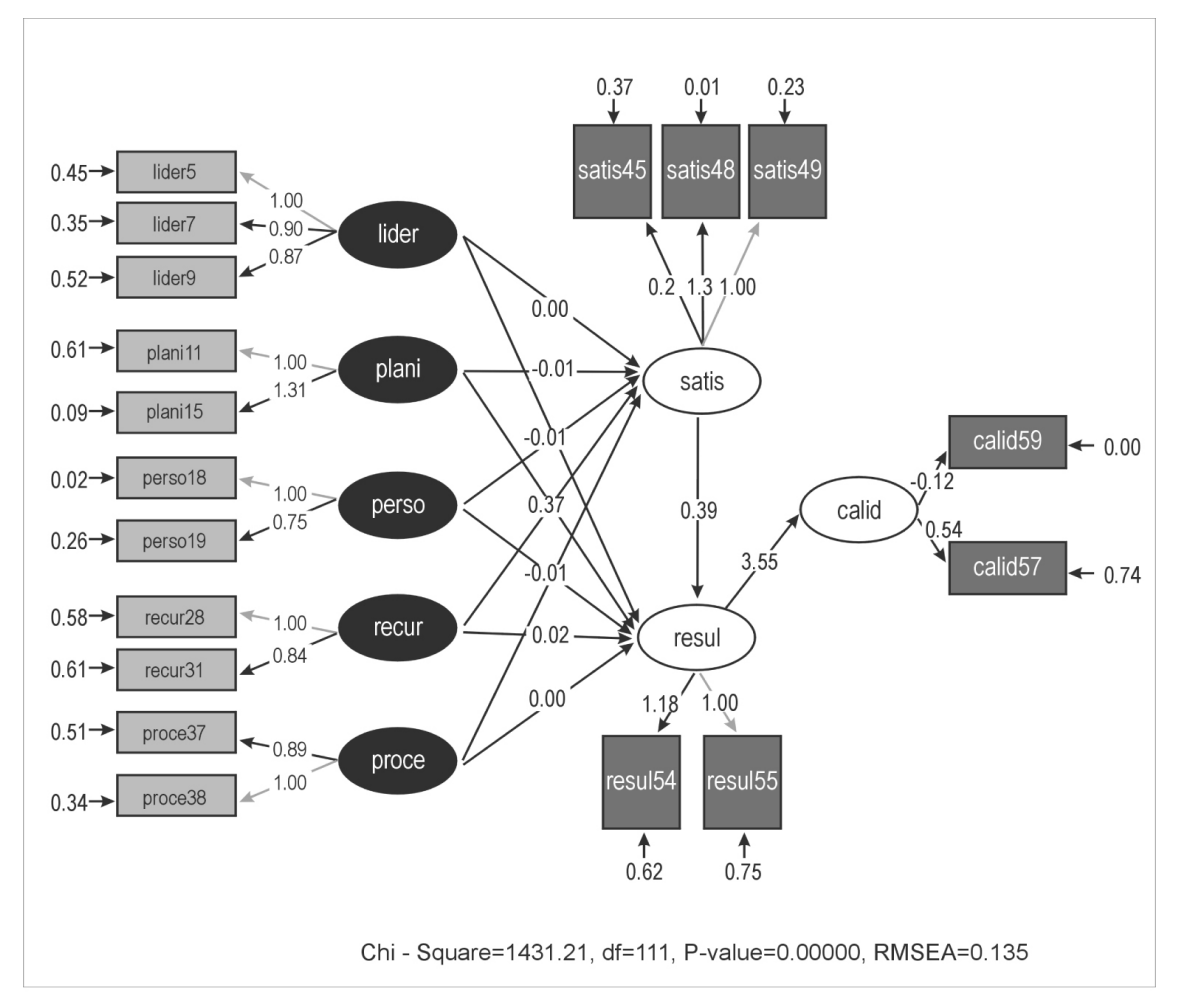

Figura 5: Modelo causal inicial con variables latentes endógenas y exógenas.

Se puede observar que el ajuste es positivo en todos los índices obtenidos para el modelo que agrupa en siete variables latentes los trece indicadores. En este sentido, todos los índices CFI, NFI, GFI y $\chi 2$ Normado, muestran valores considerados muy buenos. Finalmente se realizó un análisis al modelo con diferentes submuestras, tanto por separado como por medio del análisis multimuestra.

\begin{tabular}{|c|c|c|c|}
\hline CFI & NFI & GFI & $\chi^{2}$ Normado \\
\hline .995 & .993 & .978 & 1,91 \\
\hline
\end{tabular}

\section{Conclusiones}

En este artículo, se describen los resultados de un estudio, que a partir de la revisión teórica y de los resultados, analiza un conjunto de variables asociadas a la gestión escolar desde la perspectiva del profesorado que se estima como hipótesis, tienen efecto sobre la calidad educativa. Por ejemplo, las dimensiones planificación, gestión de personas, recursos, procesos, satisfacción, resultados y el liderazgo.
Desde esta perspectiva en el estudio encontramos efectos del liderazgo y de la gestión de personas sobre la satisfacción. En conjunto ambos factores explican un 90,1\% de la varianza. Por otro lado, las variables satisfacción, liderazgo, recursos y procesos sobre los resultados, explican un $89,1 \%$ de la varianza. Finalmente los efectos de las variables liderazgo, recursos, procesos y resultados sobre la calidad educativa explican un 94,3\% de la varianza.

Los resultados indican que en los centros educativos en que se percibe una gestión con un marcado acento en un liderazgo escolar, el compromiso de los directivos para el desarrollo con el establecimiento y el reconocimiento de la labor docente, son valorados por los profesores como positivos en la percepción de la calidad educativa.

12. Estimaciones sin sentido o teóricamente inconsistentes. Las estimaciones infractores más habituales son las varianzas de error negativas, coeficientes estandarizados que exceden o están muy cerca de 1,0 o errores estándar muy elevados. 


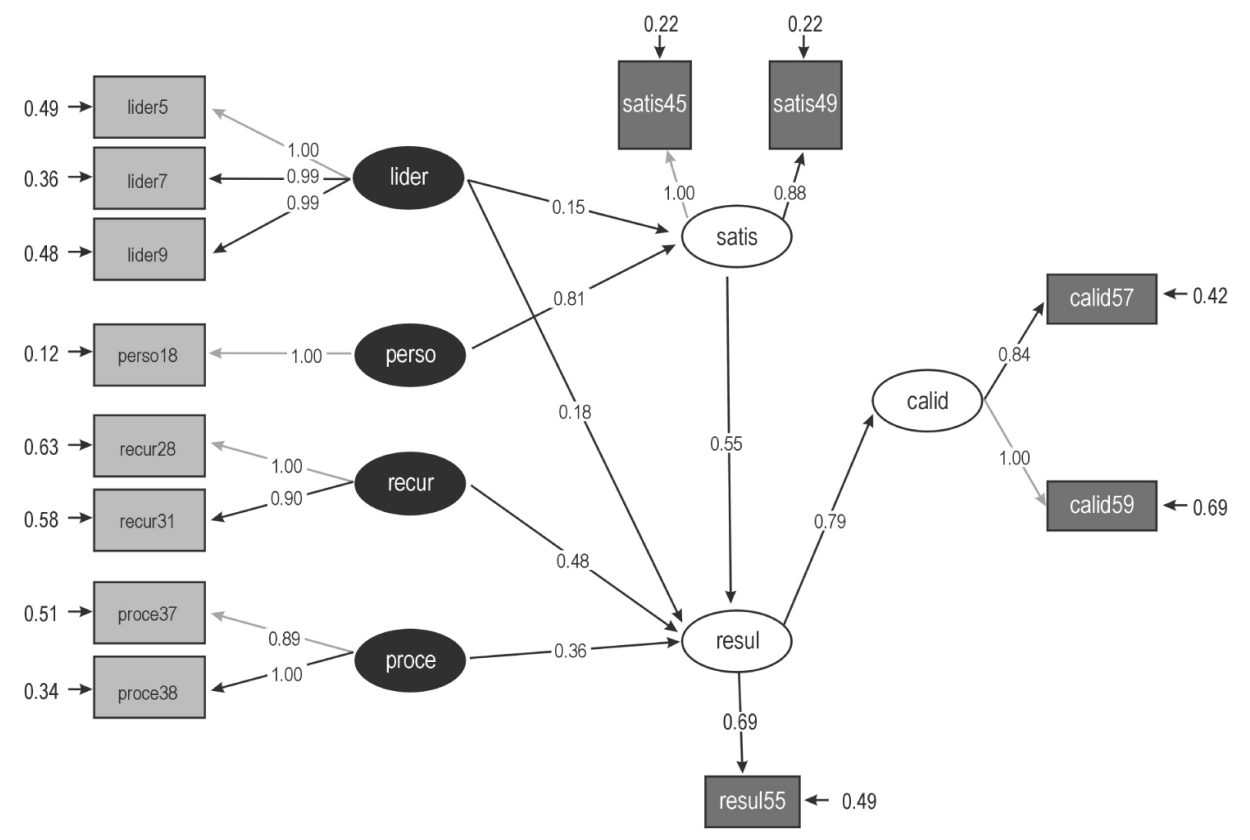

Chi - Square $=81.37, \mathrm{df}=46, \mathrm{P}$-value $=0.00101, \mathrm{RMSEA}=0.034$

Figura 6: Modelo causal resultante.

El modelo estudiado también entregó evidencia relacionada con las principales tendencias de los estudios sobre liderazgo escolar, en las cuales se modela el efecto indirecto del liderazgo y otras variables asociadas a la gestión escolar como un encadenamiento de influencias que implican enfatizar objetivos académicos, llevar un seguimiento del desempeño de los profesores e incentivar el logro de los aprendizajes en los estudiantes.

Actualmente, más que nunca, se requiere rigurosidad y sistematicidad en todo el quehacer orientado a mejorar la calidad de la educación. Los resultados de este estudio sugieren profundizar en el conocimiento de las dinámicas de influencia e interacción entre sus principales involucrados: directivos docentes, profesores y estudiantes, ya que de acuerdo al modelo causal final (Fig. 6) el liderazgo influye sobre la satisfacción y los resultados, logrando por medio de esta última variable, influir en la calidad educativa.

Lo anterior confırma lo señalado por diversos estudios respecto al liderazgo que se ejerce en un centro educativo, lo que es, sin lugar a dudas, una de las variables más relevantes que influyen en el logro de los objetivos de la organización afectando además de manera indirecta, por medio de las interacciones que se producen en los diversos actores, el aprendizaje de los estudiantes e impactando así la calidad de la educación. 


\section{Referências bibliográficas}

ALBRECHT, K. Servicio al cliente interno. Barcelona: Paidós, 1995.

BECKHARD, R.; PRITCHARD, W. Lo que las empresas deben hacer para lograr una transformación total. California: Norma, 2000.

BENTLER, P. M.; DUDGEON, P. Covariance structure analysis: statistical practice, theory and directions. Annual Review of Psychology, USA, v. 47, p. 563-592, feb. 1996.

BROWNE, M. W.; KUDECK, R. Alternative ways of assesing model fit. In: BOLLEN, K. A.; SCOTT, L. (Eds.). Testing structural equation models. Newbury Park, California: Editorial Sage Publications, 1993. p.136-162.

CANTON, I. M.; GAZÏEL, H.; WARNET, M. La calidad en los centros docentes del siglo XXI: propuestas y experiencias prácticas. Madrid: La Muralla, 2000.

CASASSUS, J. Problemas de gestión educativa en América Latina. In: CONGRESO NACIONAL REDUC, Santiago, 2000.

CEA, M. A. Análisis multivariante: teoría y práctica en la investigación social. Madrid: Síntesis, 2002.

CORONEL, J. M. Liderazgo pedagógico: un reto y una posibilidad para la mejora educativa.In: CONGRESO INTERNACIONAL SOBRE DIRECCIÓN DE CENTROS EDUCATIVOS, 5., Actas... Bilbao: Universidad de Deusto, 2008. p. 337-357.

FUNDACIÓN CHILE. Un desafío y una oportunidad para el mejoramiento de la educación chilena. In: PROGRAMA DE CERTIFICACIÓN DE LA CALIDAD DE LA ESCOLAR GESTIÓN, Santiago de Chile. 2003.

FUNDACIÓN EUROPEA PARA LA GESTIÓN DE CALIDAD. Modelo Europeo (EFQM) de excelencia. Bruselas. 1999.

GEIJSEL, F.; SLEEGERS, P.; VAN DEN BERG, D. El liderazgo transformacional como condición que favorece la implementación de programas de innovación a gran escala en las escuelas: perspectivas del profesorado. In: CONGRESO INTERNACIONAL SOBRE DIRECCIÓN DE CENTROS EDUCATIVOS, 3., Actas... Bilbao: Universidad de Deusto, 2000. p. 379-414.

HAIR, J. et al. Análisis multivariante. Madrid: Prentice Hall Iberia, 2001.

JORESKÖG, K. G. A general approach to confirmatory maximum likelohood factor análisis. Psychometrika, New York, v. 34, n. 2, p. 183202, jun. 1969.

JORESKÖG, K. G.; SORBÖM, D. LISREL 7: a guide to the program and applications. Chicago: SPSS, 1989. LISREL 8: new statiscal features. USA: Editorial Scientific Sofware International, 1993.

LEPELEY, M. T. Gestión y calidad en educación. Santiago de Chile: McGraw-Hill. 2001.

PEREZ JUSTE, R. La calidad de la educación. In: PÉREZ JUSTE, R. (Org.). Hacia una educación de calidad, gestión instrumentos y evaluación. Madrid: Narcea, 2001. p. 13- 43.

LÓPEZ RUPEREZ, F. Gestión de calidad y mejora escolar. In: PÉREZ JUSTE, R. (Org.). Hacia una educación de calidad, gestión instrumentos y evaluación. Madrid: Narcea, 2001. p. 45-73.

MARCHESI, Á.; MARTIN, E. Calidad de la enseñanza en los tiempos de cambio. Madrid: Alianza, 1998.

MAUREIRA, 0. C. Avances del liderazgo directivo. En: CONGRESO INTERNACIONAL SOBRE DIRECCIÓN DE CENTROS EDUCATIVOS, 5., Actas... Bilbao: Universidad de Deusto, 2008. p. 319-335.

MINISTERIO DE EDUCACIÓN. Calidad en todas las escuelas y liceos: sistema de aseguramiento de la calidad de la gestión escolar. Chile: Publicación de la Unidad de Mejoramiento Educativo, División de Educación General, 2005.

RUIZ OLABUÉNAGA, J. Sociología de las organizaciones. Bilbao: Universidad de Deusto, 1995. 
SENGE, P. M. La quinta disciplina: el arte y la práctica de la organización abierta al aprendizaje. Barcelona: Granica, 1992.

Recibido en 10.11 .09

Aprobado en 04.08.10

Pablo López Alfaro es profesor tarea completa en la Facultad de Ciencias Sociales, Departamento de Educación, Universidad de Chile. Licenciado en Física, Doctor en Educación. 